\title{
SOME OBSERVATIONS ON THE PUNCTATE KERATIDES IN AFRICA*
}

\author{
BY \\ F. C. RODGER \\ Director, West African Ophthalmic Survey $\dagger$
}

THE "keratitis punctata superficialis" described by Fuchs (1889) is better known to-day as epidemic virus kerato-conjunctivitis. (The nummular keratitis of Dimmer is almost certainly a variant of it.) It has been reported in epidemic form from Europe, America, Tasmania, India, China, Japan, Java, Malaya, Hawaii, and Canada, and in all these countries sporadic cases are the rule. The position has been excellently reviewed by Hogan and Crawford (1942). The disease was found to be so common during the course of a recent ophthalmic survey carried out in British West Africa that there can be little doubt it is widespread in the African continent also. In this part of the world, however, the differential diagnosis is complicated by certain tropical diseases, especially onchocerciasis, all of which have a serious prognosis. Because of this, the present study was started in the hope of finding some clear-cut differences in the clinical pictures to help in diagnosis.

\section{Different Types of Punctate Keratides}

The clinical picture of epidemic kerato-conjunctivitis is well described by Cockburn (1954). A claim was made that the causative virus was isolated by Sanders and Alexander (1943), but it has recently been suggested that the aetiological agent is Type 6 of the group of adenoid viruses (Jawetz, Kimura, Nicholas, Thygeson, and Hanna, 1955). It should be noted that trauma increases the susceptibility of the eye to infection (Viswalingam, 1941). While the characteristic lesion is punctate in form, involving Bowman's zone, coalescence may lead to larger opacities of variegated shapes and their appearance in the interstitial stroma is by no means rare. Relapses are common.

An acute punctate kerato-conjunctivitis closely simulating the epidemic type is the most common manifestation of herpes febrilis keratitis. The well-known dendritic keratitis, which the writer found very common in India, was seen only twice in some 5,000 cases of eye disease examined in Africa. Disciform keratitis also appears but rarely. Characteristically, the punctate

* Received for publication February 2, 1957

† Sponsored by the British Empire Society for the Blind. 599 
lesion is recurrent and associated with fever. Corneal anaesthesia is an invariable accompaniment. Infection of the cornea is also said to be facilitated by minor epithelial injuries. Fluorescein stains the lesions only early in their course. This picture, apart from corneal anaesthesia, is clinically identical with the epidemic virus keratoconjunctivitis, even as far as the distribution and appearance of the opacities go.

Punctate corneal opacities of the same type occur in the tropics in trachoma, leprosy, and onchocerciasis. Trachoma and leprosy may be quickly dismissed, because in the first disease the opacities are always associated with an active and unmistakable pannus trachomatosus, and in the second they are accompanied by the easily diagnosed signs of either nodular or anaesthetic leprosy, especially the latter. Superficial punctate keratitis is not the most common feature of ocular leprosy in Africa nor-and in this we are in agreement with Barros (1939) - is corneal anaesthesia a prominent finding when the keratitis is present. Apart from the obvious cutaneous signs of the disease, leprous opacities do not stand out in a clear cornea as in the epidemic cases, but lie within a grey veil of corneal infiltration as in trachoma. Moreover the opacities are very small $(0.25 \mathrm{~mm}$.). For these reasons the pictures should not readily be confused.

The one tropical disease which has been said exactly to simulate virus kerato-conjunctivitis is ocular onchocerciasis. Luna (1919) first described a chronic form of punctate keratitis which he unhesitatingly ascribed to the activities of the parasite, claiming that the picture was specific, although admitting that the corneal lesions were somewhat similar to those described in Europe by Fuchs. Luna's description showed the lesion to commence with acute photophobia and lacrimation and a smarting pain, which soon settled. The lesion then ran a chronic course. Nodules were present, and on this evidence alone the condition was considered to be due to onchocerciasis. His view was lent much support, however, by his claim that subsequently the classic picture of an onchocercal anterior uveitis supervened.

Hissette (1937) stated that he does not agree with Luna that this lesion is specific. He considers it to be in all respects similar to other types of secondary punctate keratitis. He mentions leprosy and trachoma as examples of the latter, the punctate corneal lesions being classified as "epiphenomena". At the same time, he recognizes the existence of many cases of a primary punctate kerato-conjunctivitis in Central Africa, similar, he says, to those described by Herbert and Kirkpatrick in India. He classifies the punctate keratitis of Fuchs as secondary, on the grounds that the lesion spreads from the conjunctiva. The epidemics described by Fuchs (1889) and by Herbert (1901) and Kirkpatrick (1920), nevertheless, are now all known to be classical examples of epidemic virus keratoconjunctivitis. The value of Hissette's somewhat involved arguments lies in his later statement that with the slit lamp he could see microfilariae dying 
in the cornea, and exudations forming around them. He does not enlarge on this.

Ridley (1945) is another who observed (on one occasion) an opacity forming around a dead microfilaria in the cornea. He states that the lesion is specific and primary. He describes the opacities as lying below Bowman's membrane in the stroma in the lower half of the cornea, especially nasally. Their size, he says, is about $0.6 \mathrm{~mm}$. in diameter, their colour is grey, and they are nummular in shape, with ill-defined edges. Within recent lesions, according to this worker, the dead body of a microfilaria may be seen (presumably by slit lamp) lying straightened out in a horizontal direction. The appearances of the two corneae, Ridley goes on to say, are usually strikingly similar. The whole condition is a quiet one, without signs of corneal vascularization. In short, Ridley shows the punctate keratitis of onchocerciasis to be an avascular nummular interstitial keratitis, the origin of which depends upon the formation of opacifications around dead microfilariae. This is the generally accepted view to-day. As such, there appears to be very little difference indeed between this clinical picture and those of the punctate keratitis due to the herpes and the epidemic viruses, except in the level at which the opacities are found. Here Ridley disagrees with Hissette, who believes them to lie on either side of Bowman's membrane, and the present writer confirms Hissette's observations. As, after all, the distinction is one of a very fine degree only, it would seem that some other argument must be found if these three conditions are to be distinguished one from the other.

In passing, Greeley disease should be mentioned. This lesion, described recently by Cockburn (1953), is a punctate keratitis associated with muscle pains and pharyngitis. It may prove in the end to be due to a variant of the epidemic-type virus. To date no other cases have been reported.

\section{Clinical Description of the Three Main Types of Punctate KERATIDES SEEN IN 750 AFRICAN SUBJECTS}

Of 750 cases of eye disease (of mixed origin) in an endemic onchocerciasis area, 300 had microfilariae in the conjunctiva and 450 had none.

Of the 450, 302 had positive skin biopsies, and the other 148 did not suffer from onchocerciasis at all.

Of the 300 with positive conjunctival biopsies, 160 had corneal lesions due to onchocerciasis; of these 160, 22 exhibited punctate keratitis associated with onchocercal sclerosing keratitis, and 27 with a limbitis. Of the 140 with positive conjunctival biopsies but no signs of onchocercal keratitis, 33 exhibited corneal opacities.

Of the 302 with positive skin biopsies but negative conjunctival biopsies, twelve exhibited corneal lesions associated with corneal opacities, and thirty had corneal opacities alone. Of the 148 who did not suffer from 
onchocerciasis, 29 exhibited corneal opacities, none being associated with any other corneal lesion.

It may be assumed from these figures that the 22 cases of corneal opacity associated with a characteristic onchocercal sclerosing keratitis should illustrate features typical of the punctate keratitis of onchocercal origin and may be compared with the 29 cases not suffering from onchocerciasis. One is still left of course with those cases of corneal opacity in which microfilariae are present in the conjunctiva but are not associated with any other sign of onchocercal ocular disease; they will be considered later.

(a) Punctate Keratitis of Onchocerciasis.-It is possible, although perhaps theoretically unlikely, that an avascular nummular interstitial keratitis (Ridley's term) may occur in the absence of other ocular lesions. The parasites enter the-cornea at the limbus, usually from the sub-conjunctival space, and one would expect them to produce changes either in the conjunctiva or at the limbus before or simultaneously with any change appearing in the cornea. Neither Ridley nor Hissette mention this, although it must be said that most of the excellent drawings of the latter illustrate it. This has to be emphasized, in our view, right at the outset. The appearance of these corneal opacities associated with the characteristic sclerosing keratitis of onchocerciasis ( 22 cases) was as follows:

Their size varied greatly from 0.25 to $2 \mathrm{~mm}$. in diameter. Their colour was usually densely white or yellow. They did not stain. Their position in the optic section was nearly always the same: they invariably invaded Bowman's zone as well as the lower layers of cells in the epithelium; in advanced cases they were found at a deeper level in the stroma, though this was rare and the term "superficial" appears to be preferable. The opacities were round or polyhedral in outline. They were situated in close relation to the protruding tongues of pannus onchocercosus, fairly frequently in one eye only; as the pannus was almost always found in the lower half of the cornea, usually commencing at 3 and 9 o'clock, the punctate opacities sometimes intruded on the pupillary area; we seldom saw them in the upper cornea. As might be expected they were accompanied by lacrimation and photophobia; the discharge was clear and watery. There is some doubt in our mind whether these opacities originated as a result of reactions around the dead bodies of microfilariae, for we have no histological evidence of it; on several occasions, such opacities were excised and stained, but no microfilariae were found; using a very high magnification we have on occasion seen living microfilariae moving in the vicinity, but that is the most we can say. (This will be discussed later.) The acute onchocercal keratitis associated with punctate opacities frequently quietens down without treatment, and there may be some consequent clearing of the opacities; but, even when such a state of affairs exists in a quiet eye, the condition is usually easy to diagnose, as the pannus onchocercosus seldom resolves to any extent at all; it is more difficult to be certain in the case of a limbitis, as this frequently resolves quite considerably. In the active stage, however, there is little doubt. 
(b) Epidemic Type of Punctate Keratitis.-The characteristic picture in Africa of this condition was observed over an 18-month period in eleven of the 29 subjects exhibiting this lesion within the main group of 750 cases. None of these patients was suffering from onchocerciasis. At its simplest, the condition showed itself as an acute hyperaemia of the conjunctiva, followed in a few days by lacrimation, and after a few days more by the appearance of the punctate opacities. Lacrimation was the rule, and photophobia very common; the discharge was usually clear and watery, although in one-fifth of the cases it was sticky and white at some stage in the illness. No distinctive bacillus-such as Morax-Axenfeld-was isolated. The discomfort was invariably bilateral, although the appearance of the punctate opacities was not infrequently restricted to one eye. A mild folliculosis and a slight chemosis of the lower fornix occurred in one-third of the cases, but was transient and lasted only about a week. The feeling that a foreign body lay under the lids was usual, and a sore throat was occasionally a symptom. Defective vision, when noted, was always due to invasion of the pupillary area of the cornea by the opacities. The attacks were always fairly mild. (The most severe attacks were seen in a few expatriate Europeans apart from the members of this group.) The characteristic sensation was one of discomfort, not pain. After the acute phase, which lasted from 2 to 3 weeks, the condition settled down to a long period of chronicity, punctuated by slight relapses, at something like 3-monthly intervals in the majority of cases. In its chronic phase, slight lacrimation alone was observed; however, with interference at examination, a hyperaemia of the bulbar conjunctiva quickly commenced; in short, the conjunctiva was readily irritated. Some cases healed spontaneously within a year; others were still active at the end of 18 months.

Objective examination revealed that a mild degree of hypo-aesthesia existed in the region of the opacities, but this was never as complete nor as constant as that which occurred in herpes febrilis keratitis. At no time did we observe conjunctival haemorrhages nor membrane formation. Preauricular adenitis occurred in most cases, but as this is very common in Africa (because of lice, scabies, etc.) little diagnostic significance can be attached to it. From the start of these studies, specific descriptive terms for the different appearances of the corneal opacities were used, with a view to discriminating between possible stages in their development from onset to resolution and between the punctate opacities of the epidemic type of lesion now under discussion and those associated with onchocerciasis and herpes febrilis. Only the three most common will be described below.

The newly formed opacity, from $0.25 \mathrm{~mm}$. to $1 \mathrm{~mm}$. in diameter, looks like a "morula", that is to say the opacity consists of a round collocation of regularlyspaced grey flecks; in its last appearance the opacity is of the same diameter but has become uniformly grey in colour; it is like a "patch". Several intermediate variations were seen. Over the 18 -month period of the observations, recovery 
was invariably shown to be accompanied by a shift from the morula type to the patch type. Many such patches were subsequently seen to diminish in size, usually clearing first in the centre to form a "halo", so it seems likely that of those finally disappearing all went through this morphological change. This helps in the prognosis. It takes about 6 months for this to happen and about another 3 for the subsequent patch to fade if it is going to fade. Where opacities remain after a year, it seems they are most unlikely ever to disappear, even if the configuration has changed from morula to patch. Where the number of opacities was great (forty or sixty) in a severe attack, they had a distinctive appearance. They were smaller, $0.25 \mathrm{~mm}$. on average, and are best described as looking like asterisks. In fact, they could be seen under high magnification to be small morula-types. They were yellowish-white and apparently the colour never altered, even when they were found to be diminishing in size before disappearing. One cannot help wondering if this picture may not be caused by a more virulent strain of virus.

As a rule, there were seldom more than six opacities in any one cornea; frequently there was only one. They were most frequently situated centrally or paracentrally, but, where the number was great, they spread over the entire cornea. All cases in the present group which had more than three attacks in a year showed an increase in the number of opacities when seen at the end of the period of observation. This, of course, to some extent depended upon the date of the last relapse or attack. In only three of the eleven cases examined did all the opacities present disappear; persistence is therefore to be expected, the average number of persistent opacities in the present study being three divided between the two eyes. This picture was seen time without number in healthy African eyes outside the main group of 750 in this paper, thereby giving us some idea of how widespread the disease is.

The corneal opacities are usually situated in Bowman's zone. As a result, there may be permanent damage to the superficial surface of the corneal stroma. Seen by the ocular biomicroscope, this zone forms the second relucent line, and must be regarded as part Bowman's membrane, part superficial corneal stroma. It is not surprising, therefore, that residual opacities are so common. The opacities were also found on occasion lying entirely in the epithelium or in the interstitial layers deep to Bowman's zone. Picked out quickly by the method of sclerotic scatter, they can be localized best in optic section by means of a very narrow beam. The larger ones were often seen to be saucer-shaped. The opacities nearly always remained isolated, and it was only rarely that fusion of two or three adjacent ones was observed. They were not always exactly round. None of them stained with fluorescein, which suggests that the health of the epithelium is not appreciably lowered. While only eleven such cases were observed over a period of 18 months, 56 other acute cases were noted in our later records, the clinical appearance of which agreed in every particular. This was also true of another group of thirty cases followed over a period of 9 months.

(c) Punctate Keratitis of Herpes Simplex.-It has already been said that the dendritic form of this disease is extremely rare in Africa. The disciform manifestation of herpes simplex (or febrilis) keratitis cropped up at regular 
intervals during the course of the survey. By far the most common manifestation, however, is the punctate kerato-conjunctivitis, which at first appearances looks so like the epidemic type. Eighteen cases were examined over a 15-month period, and many others were observed for shorter periods during the survey. While it is fundamentally true to say that the two virus keratides present identical clinical pictures, there nevertheless emerged in all our herpes febrilis cases two constant factors not present in the epidemic type of punctate keratitis.* The first of these was the presence of an almost constant corneal anaesthesia, the second was the presence of at least one larger polyhedral opacity among the other opacities. In the epidemic type it is a hypo-aesthesia rather than an anaesthesia, and some times not even that; the corneal opacities moreover are always nearly round. These two findings, taken in conjunction with the history of an initial attack of fever (not reported clearly in every case, one must admit), make a diagnosis of herpes febrilis keratitis very probable. Where the diagnostic demonstration of increasing antibody titre during the first weeks of the illness is impossible, it must be admitted that even such minor diagnostic pointers are of some importance. Unlike the epidemic type, new attacks were common. The position of the opacities in the beam was identical for the most part with those seen in the other two diseases, except that deeper interstitial opacities (outside Bowman's zone) were never noted.

\section{Discussion of the Aetiology of Isolated Corneal Opacities IN THE QUIET EYE}

The problem posed by the presence of a few "morula", "halo", or "patch" type corneal opacities in a quiet eye, despite what has been said, remains. True, in the absence of nodules, of positive conjunctival and skin biopsies, of any evidence of atrophy of the skin, and of residual ocular onchocercal lesions, onchocerciasis as an explanation is unlikely. Even where the conjunctival biopsies contain parasites in a quiet eye, nevertheless, the observer is no further forward. It must be remembered that, in the present survey, the virus punctate keratides were very common in endemic onchocerciasis areas.

As we have seen, out of 148 subjects not suffering from that disease in an endemic onchocerciasis area, eleven exhibited the punctate keratitis of the epidemic type and eighteen exhibited herpes febrilis keratitis. Where the opacities are due to onchocerciasis, the whole condition may settle down and some resolution occur; in this event the final appearance may simulate that due to the two virus diseases. Where then, as has been said, the conjunctival biopsies are positive, and punctate opacities are present, even with co-existing evidence of an onchocercal sclerosing keratitis, one cannot say dogmatically that the punctate opacities have been due to any one of the three lesions under discussion. The high incidence of the virus keratides

\footnotetext{
* The average size of the opacities in recent epidemics due to the adenoviruses appears to have increased,
approximating even more closely to that found in herpes febrilis.
} 
in Africa is more convincingly illustrated by our observations in non-endemic areas; in a group of 120 Africans attending a teacher's training college, 41 were found to have corneal opacities of the type under discussion. None of them had onchocerciasis. Sixty per cent. of those with opacities had a history of an attack of fever during or immediately preceding their eye trouble, and only 38 per cent. of those without opacities in the group gave such a history. In another series of 100 young men and women, 23 had corneal opacities, and only one suffered from onchocerciasis. While we have not stressed the association of enlargement of the multifibre corneal nerve bundles in herpes febrilis keratitis, inasmuch as this observation is so open to human error, such an enlargement existed in our view in one-third of the first group and in one-half of the second. Malaria is such a commonplace in the tropics that it is easy to lose sight of the fact that despite his degree of immunity, the African also suffers from it. In a questionnaire issued to the training college mentioned above, this subject was investigated, and the answer showed that on the average a young African will have two or three attacks of fever every year (2.7). When dealing with African populations, it is important to remember that relapses of malaria occur for the greater part during the rains, which fall from May to September on the West Coast. Corneal opacities due to herpes febrilis keratitis may then be expected during this period; knowing as we do that they take from 6 to 9 months to disappear (apart altogether from the fact that a few opacities usually persist and that recrudescences are the rule), it can be seen that opacities from this cause alone will be present during every month in the year. It is not surprising, therefore, that their incidence is so very high. Distinguishing the two virus types on the basis of what has been mentioned above, in six cases of corneal opacity in African subjects, three according to our records will be due to the herpes virus, two to the epidemic type, and one to onchocerciasis.

With high magnifications we have studied the activities of microfilariae in the corneal tissue on many occasions, but have never had the good fortune to observe an opacity forming around a motionless one. On two occasions a living parasite was excised within its segment of corneal tissue, and was subsequently observed under the microscope in physiological saline; both were seen to crawl between the basal epithelium and Bowman's membrane, though they found it difficult to move, having to struggle violently in forcing a passage. It has already been noted that even minor epithelial trauma may precede the virus punctate keratides, and it is not beyond the realms of possibility that activities as forceful as those observed may produce just this state of affairs; in short, even when a characteristic onchocercal lesion of the cornea exists, because of the activity of the parasites in this tissue, an attack of one or other of the virus keratides may be precipitated. The vestigial space between Bowman's membrane and the epithelium through which the microfilariae pass is traversed by the delicate end-fibres of the 
corneal nerve plexuses, which, it would seem, must be particularly vulnerable to this sort of attack. We mention this as another possible explanation of the lesions.

It has been pointed out that opacities sometimes resolve on administration of Hetrazan, and that when they appear again some months later, the conjunctival biopsies are found once more to be positive. At first sight such an association might seem a straightforward one. Theoretically, the filaricide by killing any other microfilariae in the cornea should increase rather than decrease the number of opacities, provided the pathogenesis of these lesions in the quiet eye depends upon the presence of their dead bodies, which, as indicated already, we doubt. The re-association of live microfilariae and punctate opacities certainly seems significant, and if correct must cast some doubt on the theory of the pathogenesis of onchocercal punctate keratitis.

Appelmans (1946) suggested that the latter might be an allergic reaction due to the hypersensitivity of the corneal tissue to the parasites. There is to date no evidence in support of this, apart from the belief of Taub, Miller, and Fowler (1949) that a superficial punctate keratitis (unconnected with onchocerciasis) can be attributed to allergy. On the other hand, it would seem that all the evidence points to such a lesion being typical of virus disease of the cornea. Trachoma, herpes febrilis, herpes zoster, molluscum contagiosum, even Newcastle disease, all virus infections, illustrate this point. Nevertheless, there is much to be said for the view that the punctate keratitis may occur as a result of a hypersensitive state. The widely scattered and fairly uniform distribution of the corneal opacities, so frequently described as due to onchocerciasis, is highly unlikely to have been caused by the death of parasites at each site. As a result of our work on experimental onchocerciasis, to be published later, such a large number of parasites as is postulated would almost certainly lead to a violent sclerosing keratitis. The very uniformity and equality of the distribution is against the claimed pathogenesis. How then may this reappearance of positive conjunctival biopsies with corneal opacities be explained other than by allergy?

The answer may lie in the coincidence of the periodicity of the virus keratides with the re-emergence of the parasites after Hetrazan therapy. It takes from 3 to 6 months for microfilariae to appear again in the skin after a course of $10 \mathrm{~g}$. Hetrazan, and, as already noted, our cases of virus keratitis had either relapses or recrudescences roughly at the same interval. If the latter condition is treated with Hetrazan, some resolution would be expected, one might almost say despite the drug, and a flare-up 3 or 4 months later would not be surprising.

It has been shown elsewhere (Rodger, 1957) that the Individual Density Figure* in those suffering from onchocercal kerato-uveitis ranges between

* The Individual Density Figure is an index of the density of infection in the individual, marks being given for positive biopsies taken from the conjunctiva, neck, chest, thigh, and calf of both sides, repeated when negative. 
26 and 31 . Where corneal opacities were present along with pannus onchocercosus, the mean I.D.F. was 27 ; where such opacities were present in an otherwise healthy eye, however, the mean figure was 14 although the subject was suffering from onchocerciasis. With such a low figure it is difficult to accept onchocerciasis as the cause, except perhaps in the unlikely circumstance of a head-nodule being present.

\section{Histopathology of Punctate Corneal Opacities}

The pathological findings on non-onchocercal punctate keratides reported in the literature are inconclusive and at variance. Duke-Elder (1937) quotes the mixed findings of Fuchs (1889), Nuel (1894), Verhoeff (1911), and Wright (1930). Samuels and Fuchs (1952) describe how a herpetic keratitis begins in the epithelium. It is partly attenuated, partly swollen, and in considerable disorder. The chromatin is dissociated and contracted in the centre, forming an intensely-staining eosin body, separated from the original membrane of the nucleus by an unstained zone. They call this a "herpes corpuscle". The nuclei of the basal epithelium cells no longer lie at rightangles to Bowman's membrane. The superficial layers of the stroma are in addition infiltrated by leucocytes. This histopathological picture was obtained by infecting the cornea of a single eye that had to be enucleated for carcinoma. Even here, however, the information is far from complete, since it describes the lesion at only one stage in its course.

Strong, Sahdground, Bequaert, and Ochoa (1934) underline the poverty of information on the subject of onchocercal punctate keratitis; they reproduce photomicrographs and drawings from eleven cases where tissues were excised from the eye. In none of these, however, do corneal opacities appear. This is true also of the otherwise excellent photomicrographs of Hughes (1949). Hissette (1938) stated that he sometimes found in eye sections small nests of lymphocytes and monocytes, usually placed between the epithelium and Bowman's membrane. He could not conclude with certainty that they represented punctate opacities, although he was inclined to interpret them in that way.

Such scanty evidence shows that even less is known about the histopathology of onchocercal punctate opacities than about the punctate keratitis of virus origin. We obtained a little more evidence to add to this knowledge, which is given in detail below.

In the cornea of a patient suffering from acute onchocercal kerato-uveitis (I.D.F. 31), there were two corneal opacities (Figs 1 and 2, opposite) each about $1.0 \mathrm{~mm}$. in diameter, one of the patch type and the other in the shape of a halo. They were situated on the outer margin of Bowman's zone. The segment of cornea was carefully excised without damaging the vital area. The opacities can be seen to form a depression in the epithelium through the thinning of that tissue, which is here only about $15 \mu$ in width; both opacities are entirely superficial to Bowman's membrane, which seems to be undamaged. The basal cells of the epithelium are 


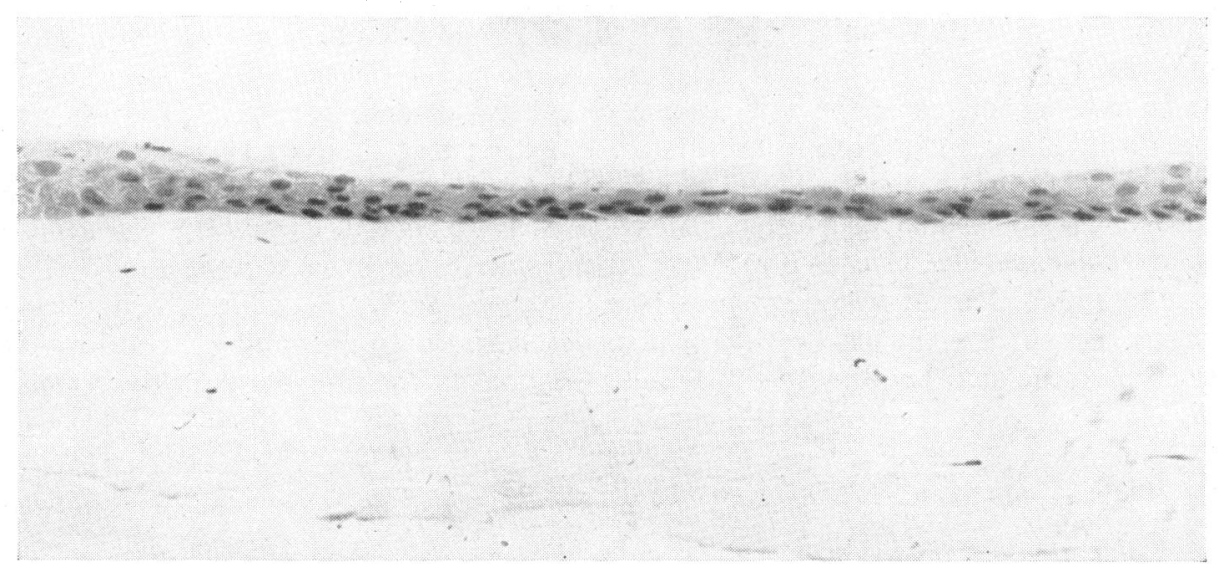

Fig. 1.-Corneal opacity in punctate keratitis of onchocerciasis. In this low-power view the full extent of the opacity can be seen. The epithelium alone is involved; it is depressed and atrophic; the nuclei are pyknotic; and the cells are flattened and in disorder. Haematoxylin and eosin. $\times 184$.

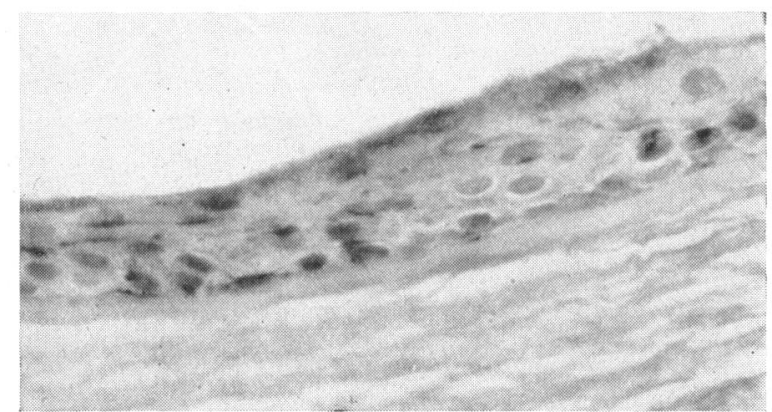

FIG. 2.-High-powered view of one end of same opacity as in Fig. 1 stained for mucin. Bowman's membrane is not affected. The mucoproteins of the basement membrane do not stain well. Nuclear pyknosis and disorder of the cells in the atrophic area can be seen. Periodic acid Schiff counterstained with haematoxylin. $\times 480$.

disordered and flattened. The nuclei are pyknotic, and the nuclear alignment horizontal. The wing cells are similarly affected. Periodic acid Schiff does not stain the mucoproteins of the basement membrane. Pigment granules have invaded the epithelium at all levels, even outside the opacity in what looks like healthy epithelium. The underlying stroma is unaffected. This picture, restricted as it is to the epithelium, does not agree with those clinical descriptions of onchocercal punctate keratitis which state definitely that the stroma and not the epithelium is involved. If this is correct, then the present case cannot be one of punctate keratitis of onchocercal origin, and it may be submitted as evidence, therefore, that an old virus punctate keratitis exists here alongside an active onchocercal kerato-uveitis. In point of fact, as mentioned earlier, the outer margin of Bowman's zone is not an unusual situation for opacities of onchocercal origin. It may be recalled that the parasites are very commonly found lying between the $39+$ 
epithelium and Bowman's membrane, which would account for this histological picture.

In the second case, the appearance of the two opacities (Figs 3 and 4) was nearly identical with those in the first case, except that their size was slightly less and they lay on both sides of the second relucent line. The subject did not suffer and had never suffered from onchocerciasis. The ocular condition was quiet. The opacities lay below Bowman's membrane, which was unaffected. The epithelium had proliferated, and its nuclei stained darkly. The basement membrane was thicker than normal, was disordered and granular, and stained heavily with periodic acid Schiff. In the area of the opacity the fibroblasts in the stroma

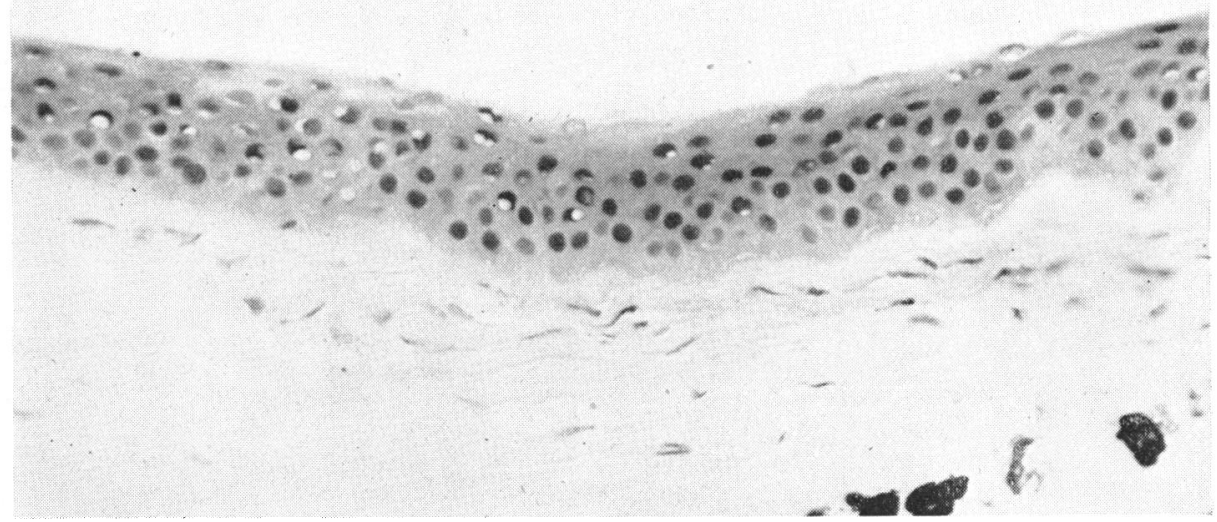

Fig. 3.-Corneal opacity in epidemic punctate keratitis. The epithelium has proliferated and its nuclei stain darkly. Bowman's membrane is irregular but otherwise unaffected. Below, in the stroma, there is an area where fibroblasts are more numerous than elsewhere. Haematoxylin and eosin. $\times 278$.

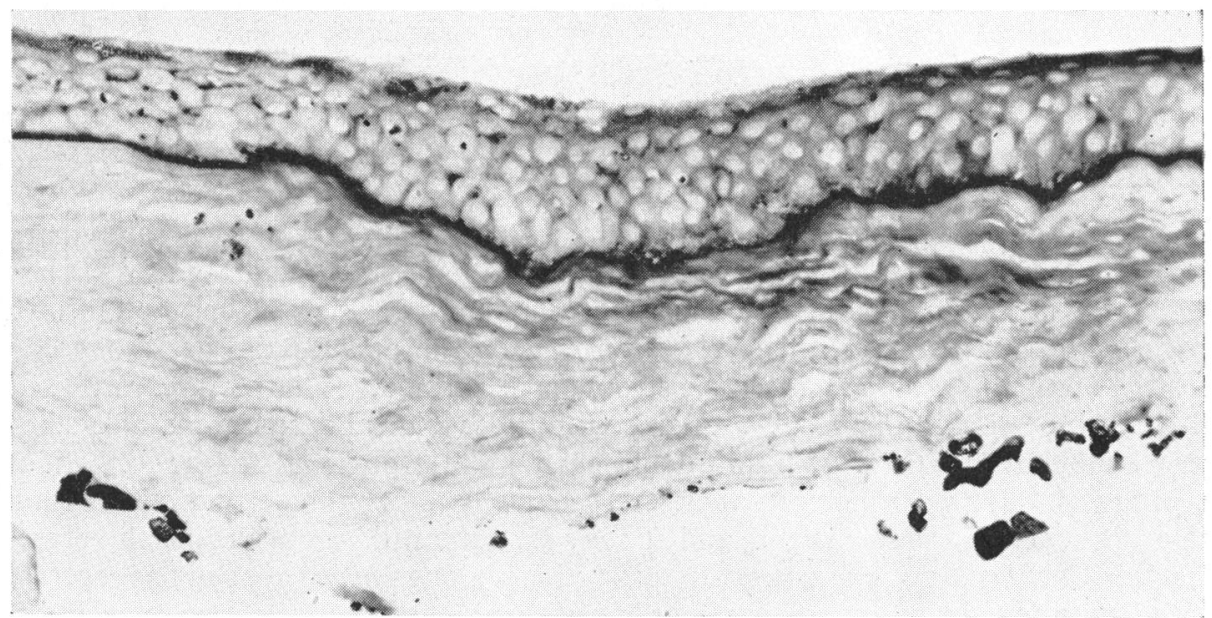

FIG. 4.-Same opacity as in Fig. 3, revealing changes in the basement membrane, which is thicker than normal and granular. The collagen fibres are disordered, and stain somewhat heavily. Periodic acid Schiff, not counterstained. $\times 278$. 
were more numerous than elsewhere. The collagen fibres were disorientated, and stained quite heavily with periodic acid Schiff. This case was diagnosed on clinical grounds as an old epidemic virus keratitis; the histological appearances are quite consistent with such a diagnosis.

\section{CONCLUSIONS}

It has been shown that the herpes febrilis and epidemic type of punctate keratitis are common in West Africa in non-endemic onchocerciasis areas, where there can be no doubt as to the diagnosis. Geographically, these areas are found next to endemic areas, so that there is no reason to suppose that the virus keratides are not also present in places where onchocerciasis exists; in fact, in patients within the endemic areas not suffering from the disease, virus keratides cases were equally common.

We believe that where the cornea is invaded by the microfilariae of onchocerca volvulus, the characteristic anterior lesions of ocular onchocerciasis will arise, and that in association with them there may appear superficial or interstitial punctate opacities of a type often slightly larger than those seen in the virus diseases and initially more dense, but in other respects similar. Where the anterior lesion is a limbitis, then complete resolution sometimes occurs, so that the end-result, a quiet eye with a few punctate opacities, may be similar to that found in the two other diseases.

Where a punctate keratitis is found to be active and severe, with many opacities scattered over the cornea but no sign of a characteristic onchocercal anterior lesion, two possibilities exist:

(1) the more likely in our view, the lesion is due to one or other of the virus keratides;

(2) there is a possibility that such a picture may occur as an allergic manifestation due to invasion of the cornea and the death of a few microfilariae (conjunctival invasion is not enough). There is no real evidence for this latter hypothesis, however. Invasion of the cornea by many microfilariae would almost certainly lead in the end to the death of several parasites, and a characteristic pannus onchocercosus would inevitably follow.

\section{SUMmaRY}

(1) The different types of punctate keratides found in the tropics are described, and the differences are stressed.

(2) The punctate keratides due to the herpes and epidemic-type viruses and those seen in onchocerciasis are shown to be somewhat similar. In a group of 750 African subjects, twenty-two, eleven, and eighteen cases of each disease respectively are described in detail. They were observed over periods of from 15 to 18 months.

(3) The aetiology of isolated punctate corneal opacities in a quiet eye is 
discussed, and it is concluded that they are seldom due to onchocerciasis, even when the conjunctival biopsies are positive. In six cases, three will be due to the herpes virus, two to the epidemic type, and one to onchocerciasis.

(4) The histopathology of two cases is given, along with photomicrographs of the lesions.

My grateful thanks are due to Sir W. Stewart Duke-Elder, K.C.V.O., for some helpful suggestions in the preparation of this paper.

\section{REFERENCES}

Appelmans, M. M. (1946). Bull Acad. roy. Méd. belg., 6 sér., 11, 428.

BARros, J. M. DE (1939). "Clinical Aspects of Ocular Leprosy”. Saõ Paulo, Brazil.

Cockburn, T. A. (1953). Amer. J. Ophthal., 36, 1534. (1954). Ibid., 38, 476.

Duke-ELDER, S. (1938). "Text-book of Ophthalmology", vol. 2, p. 1884. Kimpton, London. Fuchs, E. (1889). Wien. klin. Wschr., 2, 837.

HERBERT, H. (1901). Ophthal. Rev., 20, 339.

HissetTe, J. (1937). “Onchocercose oculaire”, Mém. Inst. roy. Colon. belg. (Sect. sci. nat. et méd., 5 , fasc. 4.

Hogan, M. J., and Crawford, J. W. (1942). Amer. J. Ophthal., 25, 1059.

HuGHES, M. H. (1949). "African Onchocerciasis". M.D. Thesis, University of Oxford.

Jawetz, E., Kimura, S., Nicholas, A. N., Thygeson, P., and Hanna, L. (1955). Science, $122,1190$.

KIRKPATRICK, H. (1920). British Journal of Ophthalmology, 4, 16.

Luna, R. Pancheco (1919). Bull. Soc. Path. exot., 12, 461.

NUEL, J. P. (1894). Arch. Ophtal., 14, 145.

RIDLEY, H. (1945). "Ocular Onchocerciasis". British Journal of Ophthalmology, Monograph Suppl. X.

RODGeR, F. C. (1957). Ibid., 41, 538.

SAmuels, B., and Fuchs, A. (1952). "Clinical Pathology of the Eye", p. 62. Cassell, London.

SANDERS, M., and AleXANDER, R. C. (1943). J. exp. Med., 77, 71.

Strong, R. P., SANDGround, J. H., BequaerT, J. C., and OCHOA, M. M. (1934). "Onchocerciasis", p. 80. Harvard University Press, Cambridge, Mass.

TAub, S. J., Miller, R. E., Fowler, M. J., and TAub, R. G. (1949). Amer. Pract. (Phil.), 3, 739.

VERHOEFF, F. H. (1911). Arch. Ophthal. (N.Y.), 40, 486.

VISWALINGAM, A. (1941). British Journal of Ophthalmology, 25, 313.

WRIGHT, R. E. (1930). Ibid., 14, 257 and 595. 